\title{
Literature Survey on Image Deblurring Techniques
}

\author{
Minu Poulose \\ Nehru College of Engineering \\ and Research Center \\ Thrissur, India
}

\begin{abstract}
Image restoration and recognition has been of great importance nowadays. Face recognition becomes difficult when it comes to blurred and poorly illuminated images and it is here face recognition and restoration come to picture. There have been many methods that were proposed in this regard and in this paper we will examine different methods and technologies discussed so far. The merits and demerits of different methods are discussed in this concern
\end{abstract}

Keywords: Face recognition, blur kernel, Point Spread Function (PSF), Local Phase Quantization, Linear Ternary Patterns,L1 norm

\section{INTRODUCTION}

Images can be generally classified into two types. They are constrained domain images and unconstrained domain images. These classifications allow the image to be considered in a restricted environment where the illumination and pose is preset. Here there is no disturbance of light and pose or any other problem and the image is ready to recognize. These types of images are known as the constrained images. But there are some images where nothing is preset. There can be illumination and posing problems. These are the unconstrained domain images. These images can be those taken with a distant camera or those images of a moving object taken with a static camera. All these images are the unconstrained images. Here there can be recognition problems. There we need to restore the image and then only we can recognize. A blurred image can be considered as a convolution function of a sharp image and a blur kernel or PSF. So in order to retrieve the sharp image we need to split the image into its blur kernel and sharp image. But the problem here is the estimation of the blur kernel. This unknown blur kernel estimation is known as the deconvolution. Most of the deblurring techniques make use of these concepts.

The rest of the paper is organized as follows: Section 2 gives a literature review on certain modern deblurring schemes highlighting its strengths and weaknesses. Section 3 compares the different schemes based on certain design criteria's. Finally concluding remarks are given

\section{LITERATURE REVIEW}

In this section we will be describing the approaches that were used in deblurring the image like subspace analysis [1], with noisy pairs [4], the general blind deconvolution methods [2], deconvolution with statistics, using local phase quantization, linear ternary patterns, set theoretic characterization etc.

\subsection{Deblurring using Subspace Analysis [1]}

Here in this method there is a training set which consists of blurred images. From this set more knowledge can be derived. Then a feature space is constructed so that the blurred faces with the same point spread function are quite similar. In the training phase, a model of each point spread function or blur kernel is computed in the feature space. For the blur kernel inference we compare a query image of blur kernel which is not known with each model and selects the closest one.
The given query image is deblurred using the blur kernel corresponding to that particular model and then it can easily be recognized. In short this algorithm, inferred PSF using learned models of facial appearance variation under different amounts of blur. Then the inferred PSFs were used to sharpen both query and target images.

This method can also be used for recognizing textual character, hand and body postures under blur. The disadvantage with this method is that it may not work well for other objects consisting of uniform texture like a plastic cup. This approach has not yet been proven for images blurred with multi unknown factors or with severe blur such as camera shake

\subsection{Blind Image Deconvolution Method [2]}

There are basically two type of deconvolution methods. They are projection based blind deconvolution and maximum likelihood restoration. In the first approach it simultaneously restores the true image and point spread function. This begins by making initial estimates of the true image and PSF. The technique is cylindrical in nature. Firstly we will find the PSF estimate and it is followed by image estimate. This cyclic process is repeated until a predefined convergence criterion is met. The merit of this method is that it appears robust to inaccuracies of support size and also this approach is insensitive to noise. The problem here is that it is not unique and this method can have errors associated with local minima.

In the second approach the maximum likelihood estimate of parameters like PSF and covariance matrices. As the PSF estimate is not unique other assumptions like size, symmetry etc of the PSF can be taken into account. The main advantage is that it has got low computational complexity and also helps to obtain blur, noise and power spectra of the true image. The drawback with this approach is of algorithm being converging to local minima of the estimated cost function.

\subsection{Deblurring with Blur Estimation Algorithm [3]}

In general the focal deblurring process is done with modeling as Gaussian low pass filtering. So the problem of blur estimation will include the estimation of the blur kernel. Here the input image (blurred) is first re-blurred by Gaussian blur kernels having different blur radius. After that the difference ratios between the different re-blurred images are used for determining the unknown blur radius. With the edge model it can be seen that the blur radius can easily be 
measured from the difference ratio and is not dependent of edge amplitude or position. The maximum of difference ratio can be seen at the edge positions. Here the advantage with this approach includes robust estimation in areas having multiple neighboring edges and this method also does not require detection of edge position and angle.

\subsection{Deblurring with noisy image pairs [4]}

In this approach the image is deblurred with the help of noisy image. As a first step both the images the blurred and noisy image are used to find an accurate blur kernel. It is often very difficult to get blur kernel from one image. Following that a residual deconvolution is done and this will reduce artifacts that appear as spurious signals which are common in image deconvolution. As the third and final step the remaining artifacts which are present in the non-sharp images are suppressed by gain controlled deconvolution process. The main advantage of this approach is that it takes both the blurred and noisy image and as a result produces high quality reconstructed image. With these two images an iterative algorithm has been formulated which will estimate a good initial kernel and reduce deconvolution artifacts. There is no special hardware is required. There are also disadvantages with this approach like there is a spatial point spread function that is invariant.

\subsection{Removing Blur with Image Statistics}

In most cases the blurred image is deblurred with a single blur kernel. But when an image having motion in different direction is considered then it can cause serious problems. As a result different kernels need to be considered. Here in this approach a single frame is considered for the whole image with the help of segmentation. It can be seen that the statistics of the derivatives are very much changed under different blur kernels. This algorithm searches for mixture model that can best define the distribution observed in the image. It results in two blur kernels and then by taking smooth layers assignment the likelihood is maximized. The output produced is a real world image with rich texture. But it has also got some limitations like the use of box filters, unknown direction of the blur, failure to describe the blur size etc. The blur patterns in real images can also turn much complex. Taking features other than simple derivative is seen improving the performance.

\subsection{Deblurring with Linear Ternary Pattern [6]}

Linearly binary patterns can be called as an extension of LBP features and are also invariant to small misalignments of pixels. This method mainly has 3 divisions. Firstly to eliminate the effects of illumination problem a pre-evaluating chain is presented without eliminating the essential features required for face recognition. Then the local ternary pattern is selected and it is less sensitive to blur effects. Here we can see that the local distance transform based on similarity is better than the local histogramming. When this method is compare with other approaches Multiscale Retinex (MSR [10]), Logarithmic Total Variation (LTV [8]) this method proves much better. So far this method has not been used along with subspace analysis. It can be incorporated to improve this method's performance.

\subsection{Using Local Phase Quantization [7]}

Phase is a property of the images which is invariant to blur. So using this property local phase quantization method has been proposed. Like the linear binary pattern used for recognition histogram of the linear phase quantization can used. It is very simple to implement and fast executing. There the challenge is the various lightning conditions. But this can be eliminated to greater extent with normalization of illumination. Here only phase information is used and so the changes are not affected. Accuracy of this method is found to be much higher than the LBP patterns. It is much better than images whose textures are not blurred.

\subsection{Face Recognition with set theoretic method [8]}

Here in the set theoretic approach both blur and illumination problem are taken into account. Instead of taking blind deconvolution as such here we can see that that the different characteristics of blur are included. Also the image is taken as a convex set. Using the Direct Recognition of blurred faces algorithm we can remove the blurring of the images. In the algorithm a sharp image gallery is blurred with a blur kernel applying different conditions. Then the distances between the blurred images are compared with the artificially blurred image and that having minimum distance is taken as the corresponding image. Followed by that the illumination challenges are taken into account. Here the illumination coefficient for image when considered at different planes are considered and is incorporated in the algorithm. Now together with the removal of blur illumination problems are also removes. It is easy to implement, not complex and returns much better result than the other previous approaches. Also here L1 norm distance is taken for making the algorithm robust to pixel misalignments.

\section{COMPARISON OF DIFFERENT DEBLURRING TECHNIQUES}

Different methods were discussed so far. To have a clear picture, see table 1.

Table 1. Comparison Table

\begin{tabular}{|c|c|c|}
\hline Method & Accuracy & Different \\
\hline SubSpace Analysis & Medium & Low \\
\hline $\begin{array}{c}\text { Blind Image } \\
\text { Deconvolution }\end{array}$ & Medium & Medium \\
\hline $\begin{array}{c}\text { Image Statistics } \\
\text { Local Phase } \\
\text { Quantization }\end{array}$ & High & Medium \\
\hline Set theoretic Approach & High & Medium \\
\hline
\end{tabular}

In the first approaches In most of the deblurring approaches it uses the most common technique called the blind image 
deconvolution. Here the unknown blur kernel is rough estimated and recognition is done on that basis. In the subspace analysis [1] the different texture blur could be easily recognized. But it also had the problem of not solving the images with uniform textures. Blind image deconvolution techniques though like a probability process if blur kernel is found correctly then it is one of the most reliable technique. Earlier techniques like using linear binary pattern, linear ternary patterns, linear phase quantization etc were used. These methods had advantages of being robust to misalignments in the pixel value.

\section{CONCLUSION}

From the above analysis we can see that though the subspace analysis [1] and blind image deconvolution [2] finds result to some extent it is prone to errors and is more or less like a probability method. In the local phase quantization technique it is accurate but not robust to different types of blurs and lighting problems can make the deblurring difficult. In the Set theoretic approach we can see that it is more accurate and different blur conditions are added on to make deconvolution method much less complex than the other approaches

\section{ACKNOWLEDGMENTS}

I extend my grateful acknowledgment to all the authors who rendered their help in the preparation of this paper. I would like to thank all my friends and well wishers whose valuable suggestion and encouragement helped for the research . Above all I am thankful to Almighty for the successful completion of my work.

\section{REFERENCES}

[1] Nishiyama, M., Hadid,A.,Takeshima,H., Shotton, J., Kozakaya, T. and Yamaguchi,O. 2011 Facial deblur inference using subspace analysis for recognition of blurred faces, IEEE Trans. Pattern Anal. Mach. Intell., vol. 33 , no. 4 .

[2] Kundur, D. and Hatzinakos, D. Blind image deconvolution revisited.

[3] Hu, H. and Haan, G. 2006 Low cost robust blur estimator Proc. IEEE Int'l Conf. Image Processing, pp. 617 - 620

[4] Yuan, L., Sun, J., Quan, L. and Shum, H.Y. 2007 Image deblurring with blurred/noisy image pairs ACM Trans. Graphics, vol. 26, no. 3, pp. 1

[5] Levin, A. 2006 Blind motion deblurring using image statistics in Proc. Adv. Neural Inform. Process. Syst. Conf pp. 841-848.

[6] Xiaoyang, T. and Bill ,T. 2007 Enhanced Local Texture Feature Sets for Face Recognition Under Difficult Lighting Conditions in AMFG 2007, LNCS 4778, pp. 168-182

[7] Ojansivu, V. and Heikkilä, J. 2008 Blur insensitive texture classification using local phase quantization in Proc. 3rd Int. Conf. Image Signal Process., pp. 236-243.

[8] Chen, T., Yin, W., Zhou, X., Comaniciu, D. and Huang, T. 2006 Total variation models for variable lighting face recognition. IEEE TPAMI 28(9), 1519-1524
[9] Vageeswaran, P., Mitra, K. and Chellappa, R. 2013 Blur and Illumination Robust Face Recognition via SetTheoretic Characterization IEEE Transactions On Image Processing, VOL. 22, NO. 4

[10] Jobson, D., Rahman, Z. and Woodell, G. 1997 A multiscale retinex for bridging the gap between color images and the human observation of scenes IEEE TIP 6(7), 965-976 